\title{
The Use of Soft Agar in the Study of Conditions Affecting the Utilization of Fermentable Substrates by Lactic Acid Bacteria
}

\author{
By R. WHITTENBURY \\ Department of Bacteriology, School of Agriculture, Edinburgh
}

(Received 21 January 1963)

\begin{abstract}
SUMMARY
Lactobacilli, streptococci, leuconostocs, pediococci and aerococci were examined in a soft agar medium which provided aerobic and anaerobic conditions in the one culture. The relation to oxygen varied in some instances with the energy source and the method by which it was sterilized. Other variations were determined by the temperature of incubation, the $\mathrm{pH}$ value of the medium and the salt concentration. Only a proportion of the organisms showed preferences or requirements for diminished aeration or anaerobic conditions. In some cases an aerobic environment was optimal or essential for the utilization of a fermentable substrate. The soft agar medium showed that the fermentation of sugars and other substances might occur as a sequel to mutation. Liquid media were compared with soft agar media; they provided little information about oxygen relationships. 'This comparison suggested several possible reasons for the variability of lactic acid bacteria reported by investigators who used liquid media in tests for fermentation.
\end{abstract}

\section{INTRODUCTION}

It is frequently stated in the literature (e.g. in Bergey's Manual, 1957), that many lactic acid bacteria are either microaerophilic or anaerobic. However, there is little definite information on this question and the organisms are frequently cultivated without regard to the possible effects of oxygen on their growth and chemical activities. A soft agar medium described below has proved to be suitable for these organisms and to give more information about their fermentative properties than do liquid media.

\section{METHODS}

Organisms. Lactobacilli, streptococci, leuconostocs, pediococci and aerococci, in all 250 strains, were examined. They were obtained from several individuals and the National Collection of Type Cultures (NCTC), the National Collection of Dairy Organisms (NCDO), the National Collection of Industrial Bacteria (NCIB), and the Institute of Applied Microbiology, Tokyo (IAм) or were isolated from fresh herbage and silage at the beginning of the investigation. An objective was to secure a set of strains in which the widest possible range of character was represented. The organisms were identified as far as possible by their physiological and morphological properties. The pediococci were classified according to Nakagawa \& Kitahara (1959).

Soft agar medium and preparation of soft agar cultures. The basal medium contained: meat extract (Lab-Lemco), 0.5 g.; peptone (Evans), 0.5 g.; yeast autolysate 
(prepared as in Gibson, Stirling, Keddie \& Rosenberger, 1958), 5.0 ml. or yeast extract (Difco), 0.5 g.; Tween 80, 0.05 ml.; agar (Davis), 0.15 g.; tap water to $100 \mathrm{ml}$. Either bromocresol purple (BCP), 1.4 ml. of a $1 \cdot 6 \%(\mathrm{w} / \mathrm{v})$ ethanolic solution, or bromocresol green (BCG), $2.8 \mathrm{ml}$. of a $0.4 \%(\mathrm{w} / \mathrm{v})$ aqueous solution, was added per l. medium. Fermentable substrates, as distilled water solutions sterilized by autoclaving at $121^{\circ}$ for $15 \mathrm{~min}$., or by Seitz-filtration, were added to the autoclaved $\left(121^{\circ}\right.$ for $15 \mathrm{~min}$.) basal medium to give a final concentration of $0.5 \%(\mathrm{w} / \mathrm{v})$ or, in the case of slightly soluble substances, $0 \cdot 25 \%(\mathrm{w} / \mathrm{v})$. The medium used in salt tolerance tests was prepared at double strength and an equal volume of $\mathrm{NaCl}$ solution was added after sterilization. Soft agar containing BCP was adjusted to

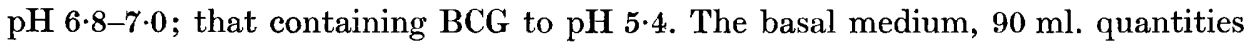
in bottles, was liquefied by momentarily autoclaving at $115^{\circ}$, allowed to cool to $48^{\circ}$ in a water bath and completed by adding $10 \mathrm{ml}$. of a fermentable substrate solution. The completed medium was replaced in the water bath or kept in an incubator at $50^{\circ}$ until required, when it was distributed in 6-7 ml. amounts to sterile $6 \times \frac{5}{8}$ in. test tubes, the amount being judged visually as the medium was poured into the tubes. A set of completed media was placed in the one rack in the water bath so that they could be inoculated with one culture. Immediately before inoculation the media were allowed to cool to $37-40^{\circ}$, a temperature which allowed ample time for inoculation before the agar set. The liquefied medium was inoculated with a drop of a turbid culture added by capillary pipette and then tilted two to three times before being allowed to set. The subsequent growth indicated that the inoculum was uniformly distributed through the medium by this procedure. When for comparative purposes substrates were autoclaved in the medium the same method of inoculation was used.

Liquid medium. This was similar in composition to the soft agar medium, but without agar. The medium was distributed in 4.5 or 2.25 ml. amounts in $5 \times \frac{1}{2}$ in. tubes. Substrate solutions, $0.5 \mathrm{ml}$. in the former instance and $0.25 \mathrm{ml}$. in the latter, were added subsequently when not included in the medium originally. Inoculation was by capillary pipette.

Inoculum medium. This was similar to the liquid medium with the exceptions that glucose, $0.5 \%(\mathrm{w} / \mathrm{v})$, was autoclaved in the medium, which was adjusted to $\mathrm{pH} 6 \cdot 5$, and the indicators were omitted.

Glucose agar. This was similar to the inoculum medium with the exceptions that agar $1.5 \%(\mathrm{w} / \mathrm{v})$ and $\mathrm{MnSO}_{4} .4 \mathrm{H}_{2} \mathrm{O} 0.01 \%(\mathrm{w} / \mathrm{v})$ were added.

Detection of hydrogen peroxide formation. Manganese dioxide agar, based on the pyrolusite agar described by Kneteman (1947), was used. Glucose agar was poured as a plate to which was added a very thin layer of glucose agar containing pyrolusite or manganese dioxide (black tech.; Harrington Bros. Ltd.), $4.0 \%(\mathrm{w} / \mathrm{v}$ ). The plates were dried and inoculated by streaking; $\mathrm{H}_{2} \mathrm{O}_{2}$ formation was indicated by clearing of the manganese dioxide under and around the growth.

Incubation temperature. Unless otherwise stated all incubations were at $30^{\circ}$.

\section{RESULTS}

The soft agar medium provided environments ranging from aerobic to anaerobic in the one culture. This was indicated by the site of growth and by the reduction of methylene blue below the top $0.5-1 \cdot 0 \mathrm{~cm}$. of the uninoculated medium. Reducing 
conditions persisted, at least in the lower half of the medium, throughout the 14-day period of incubation.

The $\mathrm{pH}$ indicators, bromocresol purple and bromocresol green, proved satisfactory in the great majority of instances. Sometimes both indicators were bleached by reducing organisms such as Streptococcus faecalis. A fourfold increase of the indicator content masked this effect and proved satisfactory in these instances. Doubtful colour changes, unaccompanied by an obvious increase in growth over that in the basal medium, were checked electrometrically.

The sterilization of fermentable substrates by autoclaving in distilled water gave the same results as sterilization by Seitz-filtration. Differences were occasionally observed, however, between growth in the presence of separately sterilized substrate and that on the same substrate autoclaved in the medium. Unless specifically mentioned, the descriptions given below apply to growth not affected by treatment of the substrates.

\section{Requirement for aerobic conditions}

Although lactic acid bacteria have been described as microaerophilic or anaerobic, a number of these organisms were found to require aerobic conditions when utilizing certain substrates. In such instances, acid-forming growth was restricted to the surface layers of the soft agar culture (Pl. 1, fig. 1). Slight, non-acid forming growth, similar to that which appeared in the basal medium, frequently developed throughout the remainder of the culture. On continued incubation, growth increased in density in the surface layers and occasionally developed as secondary disks below the surface as the medium became oxygenated. Incubation of soft agar cultures under an atmosphere of $95 \%(\mathrm{v} / \mathrm{v}) \mathrm{H}_{2}+5 \%(\mathrm{v} / \mathrm{v}) \mathrm{CO}_{2}$ confirmed that growth restricted to the surface reflected a requirement for aerobic conditions.

When substrates were utilized only aerobically in soft agar, liquid cultures occasionally gave variable results. Obvious acidity and growth were sometimes only observed after 6-8 days of incubation, in contrast to the 2-3 days required in soft agar. When acid production was weak and growth slight but none the less evident in soft agar, no acidity or only slight acidity after 10 or more days was observed in liquid cultures. Shallow shaken cultures became more strongly acid than did deep unshaken cultures.

Examples of a requirement for aerobic conditions were provided by strains of Streptococcus faecium, Pediococcus spp. and Lactobacillus plantarum utilizing glycerol; Aerococcus viridans NCTC 8251 and Pediococcus urinae-equi IAM 1684 utilizing glycerol, mannitol or sorbitol and, within the first $24 \mathbf{h r}$. of incubation, glucose; $S$. faecalis strains utilizing $m$-inositol in the absence of fumarate as an exogenous hydrogen acceptor; $L$. salivarius utilizing mannitol or sorbitol; some leuconostocs in the initial stages of hexose utilization; and strains of $L$. brevis and L. buchneri utilizing hexoses, gluconate or mannitol which had been sterilized apart from the medium.

A proportion of the organisms, therefore, required or benefited from the presence of oxygen in the utilization of certain substrates. In many cases the action on these substrates involved the formation of $\mathrm{H}_{2} \mathrm{O}_{2}$. Instances of lactic acid bacteria requiring aerobic conditions for the utilization of specific substrates have been recorded previously (Gunsalus \& Sherman, 1943; Dobrogosz \& Stone, 1959). 


\section{Requirement for anaerobic conditions}

When substrates were fermented only under anaerobic conditions, acid-forming growth was restricted to a sharply defined zone in the anaerobic part of the soft agar culture (Pl. 1, fig. 2). The height of the zone, dependent upon substrate and organism, varied from the bottom fifth of the culture to within $1.0-0.5 \mathrm{~cm}$. of the surface. Examples of anaerobic growth are some organisms of the Lactobacillus casei-plantarum group utilizing cellobiose, sucrose or glycerol, and some unclassified streptococci utilizing lactose, amygdalin or raffinose. With other substrates these particular organisms grew well throughout the medium.

Sometimes the $\mathrm{pH}$ value of the medium appeared to affect the ability of an organism to grow aerobically. Examples were observed with Lactobacillus cellobiosus and Pediococcus NCDo 1250. In an originally neutral glucose medium their initial growth and acid production occurred only in the anaerobic part (Pl. 1, figs. 3, 5) but as acid diffused upwards aerobic growth appeared, rapidly in the case of $L$. cellobiosus (Pl. 1, fig. 4), slowly in the case of the pediococcus. In soft agar initially at $\mathrm{pH} 5 \cdot 4$ both organisms grew uniformly throughout. On neutral glucose agar slopes incubated aerobically these organisms either did not grow or produced very slight growth late in the incubation period, but they grew well when the medium was adjusted to $\mathrm{pH} \mathbf{5} \cdot 4$.

Where cases of anaerobic requirements were observed in soft agar comparative liquid cultures produced variable results. Shallow liquid cultures frequently showed no acid formation whilst deep cultures frequently became acid. When incubated anaerobically, however, acid was always formed.

\section{Requirement for a diminished oxygen concentration}

In the unsupplemented basal medium many organisms produced a thin disk of growth without obvious acid formation about $0.25 \mathrm{~cm}$. below the surface. With a fermentable substance added, acid-forming growth was in certain cases confined to the same position, as for example some strains of Lactobacillus buchneri with xylose as the substrate (Pl. 1, fig. 6). In liquid cultures with the same substrates these particular organisms produced slight or questionable reactions.

\section{No strict aerobic, microaerophilic or anaerobic requirement}

In such instances growth and acidity appeared throughout the whole culture and in most cases uniformly and simultaneously (Pl. 1, fig. 7). A number of leuconostocs, however, exhibited a preference for aerobic conditions since growth and acid formation were initially confined to the surface of the medium. The later-developing anaerobic growth either became as dense as the surface growth or remained slight by comparison. In these circumstances liquid cultures gave constantly positive results.

\section{Mutant behaviour}

The formation of one or more relatively large, acid-forming colonies surrounded by slight non-acid forming growth was interpreted as mutant behaviour. Old or diluted inocula containing few viable organisms also sometimes gave rise to a few acid-forming colonies, but this could be distinguished by the use of fresh inocula and a control medium containing a substrate which was utilized uniformly by the 
organisms. The mutant colonies were frequently distributed throughout the medium (Pl. 1, figs. 8-10), but were sometimes confined to the aerobic portions ( $\mathrm{Pl}$. 1, fig. 11) sometimes to the anaerobic portion (Pl. 1, fig. 12) and sometimes to the microaerophilic region (Pl. 1, fig. 13). Occasionally only single colonies were observed in the culture (Pl. 1, fig. 14). Some organisms produced dense growth in the aerobic portion of a medium but only a few mutant colonies in the anaerobic portione.g. some strains of Streptococcus faecalis when utilizing glycerol. An unusual sequence of events was observed with a strain of the Lactobacillus plantarum-casei group when utilizing raffinose. The glucose-grown inoculum produced mutant colonies in the aerobic portion of the raffinose medium and no acid-forming growth in the anaerobic portion. The purified mutant produced dense and uniformly distributed growth in the aerobic portion of the raffinose medium and mutant colonies in the anaerobic portion. These mutants when purified gave dense, acidforming growth distributed uniformly throughout the aerobic and anaerobic portions of the medium. The $\mathrm{pH}$ value of the medium sometimes affected growthe.g. a strain of the L. plantarum - casei group grew uniformly throughout a raffinose medium adjusted to $\mathrm{pH} 5 \cdot 4$ but only 'mutantly' in the same medium adjusted to $\mathrm{pH} 7 \cdot 0$. The purified mutant grew uniformly throughout the medium adjusted to $\mathrm{pH} 7 \cdot 0$.

In all the instances of mutation mentioned, the parent cultures were purified two or three times and the tests repeated; the same behaviour was observed again. The purified mutants were subcultured four to five times through a glucose medium and compared with the parent cultures by using appropriate biochemical tests. The behaviour of the mutant and parent cultures was found to differ only on the substrate which supported mutant growth. No attempt was made to determine the nature of the mutations-e.g. whether or not utilization of the substrate was related to permeability or to the ability to metabolize it, or in the case of glycerol utilization by Streptococcus faecalis, the ability to use unidentified exogenous hydrogen acceptors of the basal medium. Fumarate was eliminated in this instance, as its addition to the glycerol medium permitted the formation of uniformly distributed, dense, fermentative growth in the anaerobic portion.

An unsuccessful attempt was made to assess mutation rates. The basal medium supported a slight amount of growth which made it impossible to be certain whether acid-forming colonies arose from mutant organisms in the inoculum or from some which developed later. Frequently $1 \mathrm{ml}$. of undiluted inoculum and $1 \mathrm{ml}$. of a 1/100 dilution of the inoculum yielded a similar number of acid-forming colonies. It was suggested by Rogosa et al. (1953) that concentration of the substrate may affect the ability of an organism to adapt to its utilization. Increasing substrate concentration up to $2 \cdot 0 \%(\mathrm{w} / \mathrm{v})$ in the present instances did not alter or mask the mutant behaviour.

In contrast to soft agar media which gave constant mutations, liquid media yielded positive results in one experiment and negative in another. In a number of trials with liquid media the proportion of positive results increased with increasing size of inoculum, and it was affected by the depth of the medium in cases requiring aerobic or anaerobic conditions and in some instances by the $\mathrm{pH}$ of the medium.

A practical aspect of these observations is their relation to the taxonomy of the organisms. If an organism is mutant on a substrate used for differentiating it 
from other species, the value of such a 'characteristic' is diminished. Such an instance was observed with leuconostocs. Three strains of Leuconostoc citrovorum, a species separated from $L$. dextranicum by its inability to form acid and dextran from sucrose, produced sucrose-fermenting mutants. These mutants could not be induced to form dextran and so remained distinct from $L$. dextranicum in this characteristic alone.

\section{The distinction between slow and weak substrate utilization}

Utilization of a substrate in soft agar was regarded as slow when the amount of growth increased over a few days and finally equalled that supported by a rapidly utilized substrate. Utilization was judged to be weak when growth remained only slightly more dense than that which developed in the basal medium and when the pH value decreased by only $1 \cdot 0-1.5$ units in a medium originally neutral, or by 0.5-1.0 unit in a medium originally at $\mathrm{pH} 5 \cdot 4$. When utilization was weak the growth was often restricted to one portion of the medium, e.g. the anaerobic portion in the case of some leuconostocs when utilizing arabinose or xylose. In a test for acid formation in a liquid medium, weak utilization tended to give an indefinite result.

\section{Effects of incubation temperature}

The soft agar medium in which glucose was autoclaved was used to test the ability of organisms to grow at various temperatures. Some strains of Lactobacillus brevis and $L$. buchneri grew uniformly throughout this medium at $30^{\circ}$, but only in the aerobic portion at $37^{\circ}$. Conversely, L. hilgardii ксIв 8040 and some unclassified streptococci and heterofermentative lactobacilli grew uniformly throughout the medium at $30^{\circ}$, but at $37^{\circ}$ only in the anaerobic portion. In a liquid medium incubated aerobically at $37^{\circ} \mathrm{L}$. brevis and $L$. buchneri showed acid formation but L. hilgardii and the other organisms did not grow. When glucose was replaced by a fermentable pentose, growth of all these organisms was uniform in soft agar at $37^{\circ}$. Another variation was noted with $L$. fructovorans NCIB 8039; at $30^{\circ}$ growth appeared uniformly throughout the medium but as the maximum temperature for growth $\left(37^{\circ}\right)$ was approached, growth and acid production became restricted to a disk about $2 \mathrm{~mm}$. in depth, $1 \cdot 0-1.5 \mathrm{~cm}$. below the surface (Pl. 2, figs. 19, 20) and did not develop outside this zone on further incubation. At $35^{\circ}$ and $37^{\circ}$, the liquid medium incubated aerobically frequently failed to support growth of this organism.

\section{Effect of different $\mathrm{NaCl}$ concentrations}

Three variations were observed in glucose soft agar. The first concerned Aerococcus viridans NCTC 8251 and Pediococcus urinae-equi IAM 1684. As the $\mathrm{NaCl}$ concentration was increased to $10 \%(\mathrm{w} / \mathrm{v})$ growth and acid production became restricted to the surface layers of the medium. A second effect observed was the great decrease in the number of organisms of the inoculum which were able to initiate growth at high $\mathrm{NaCl}$ concentrations. The growth of $P$. soyae IAM 1673 (Pl. 2, figs. 15-17) illustrates this, comparatively few colonies appearing in a medium containing $18 \%(\mathrm{w} / \mathrm{v}) \mathrm{NaCl}$. A third variation was observed with some pediococci when growing in the presence of $8-10 \%(\mathrm{w} / \mathrm{v}) \mathrm{NaCl}$; growth was then restricted to a few colonies which developed in a zone in the middle of the medium (Pl. 2, fig. 18). The manner of growth in $\mathrm{NaCl}$ media seemed to be unrelated to mutation. Transfer of 
the colonies to a medium lacking $\mathrm{NaCl}$ resulted in uniformly dense growth but when they were transferred to a medium containing the same concentration of $\mathrm{NaCl}$ the previous result was repeated.

\section{Effects of the method of sterilizing media}

The fermentable substrates were sterilized in two ways: (1) by autoclaving in the medium; (2) separately, by autoclaving or Seitz-filtration before being added to the autoclaved basal medium. The only organisms which did not behave in the same way in differently prepared media were all the strains of Lactobacillus brevis, L. buchneri, unidentified organisms closely similar to these two species, and $L$. viridescens. The growth of these organisms is described in Table 1 , which shows that with gluconate, hexoses, di- or tri-saccharides as substrates there was a difference in the two types of media. Aerobic growth was similar in both kinds of media. Anaerobically, growth developed rapidly in a medium in which the substrate had been sterilized, but slowly or not at all in a medium containing separately sterilized substrate. No attempt has yet been made to determine the reasons for these differences. It was clear, however, that a slow anaerobic development with separately sterilized substrates, when it occurred, was not a case of adaptation, since anaerobic growth was equally slow upon transfer to a similar medium. In all this work no instance was recorded of an organism being able to utilize a substrate sterilized by one method but not when sterilized by another.

\section{Table 1. Growth with substrates sterilized by two procedures}

Type of growth indicated by: aer = aerobic only; pa = aerobic growth with delayed (2-10 days) and slight anaerobic growth; a/san $=$ aerobic growth and simultaneous anaerobic growth which was at first slight; mic = microaerophilic growth; fac = growth uniform throughout aerobic and anaerobic regions of the medium; aerobic growth may finally become heavier than anaerobic growth.

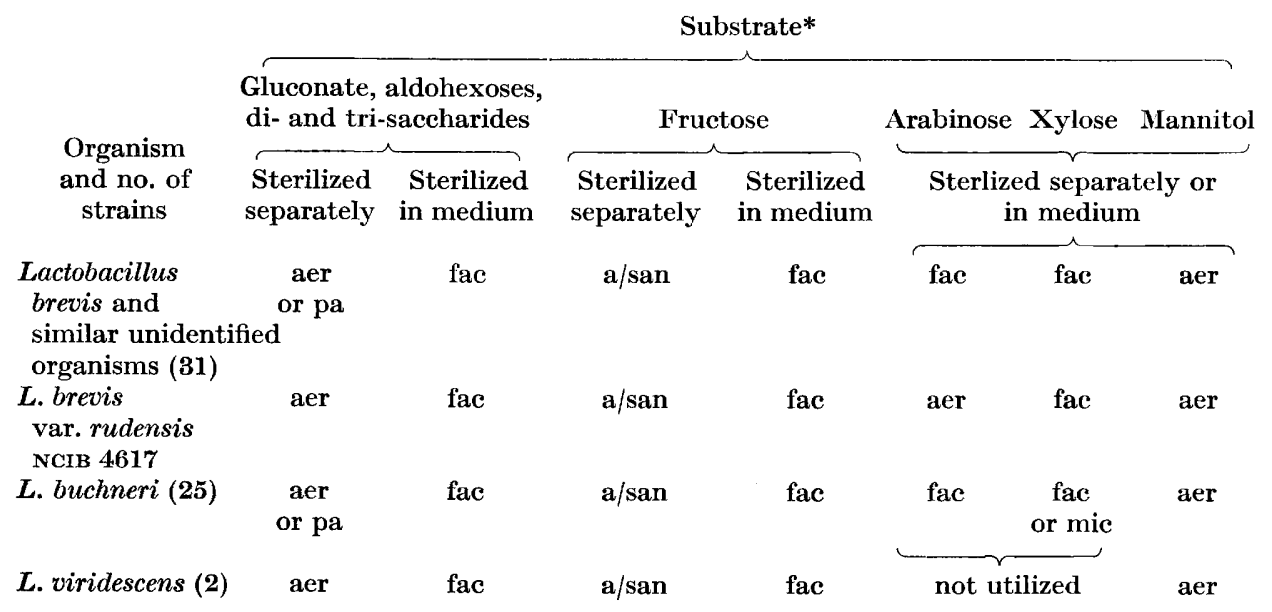

* Results apply to organisms able to utilize the energy sources specified.

Effect of citrate and manganese

When potassium citrate $(0 \cdot 1 \%, \mathrm{w} / \mathrm{v})$ was added to soft agar, the growth with separately sterilized sugars of both the strains of Lactobacillus viridescens studied, 
and of some unidentified heterofermentative lactobacilli, was greatly improved. The growth of all the other bacteria was not significantly affected. In the case of L. viridescens citrate increased the anaerobic but not the aerobic growth; with the unidentified lactobacilli, aerobic and anaerobic growth developed very slowly in absence of citrate but rapidly in its presence. When pentoses were autoclaved in a medium to which citrate had been added, the anaerobic growth of many strains of L. brevis and $\boldsymbol{L}$. buchneri was either completely or partially inhibited. Other organisms were not affected in this way.

The addition of $\mathrm{MnSO}_{4} \cdot 4 \mathrm{H}_{2} \mathrm{O}(0.01 \%$, w/v) to sugar-containing soft agar resulted in a marked increase in the amount of aerobic growth of all the strains of $L$. brevis, L. buchneri, unidentified organisms closely similar to these two species and $L$. viridescens. The anaerobic growth of these organisms did not appear to benefit from the presence of manganese. The growth of the other bacteria studied was not obviously improved by adding manganese to the media.

\section{$A$ hydrogen peroxide effect}

A number of leuconostocs exhibited a preference for aerobic conditions when utilizing glucose and related compounds. In a soft agar medium containing one of these sugars, aerobic growth was frequently separated from the later-developing anaerobic growth by a growth-free zone (Pl. 2, fig. 21) which often diminished on prolonged incubation ( $\mathrm{Pl}$. 2, fig. 22). The addition of catalase to the medium prevented the occurrence of the growth-free zone as did manganese dioxide, which was dissolved in that area. This indicated that $\mathrm{H}_{2} \mathrm{O}_{2}$ was being formed at the surface, and, diffusing downwards, it inhibited the initiation of anaerobic growth.

\section{DISCUSSION}

The soft agar medium proved to have several advantages over liquid media in the study of substrate utilization by lactic acid bacteria. Incubated aerobically, soft agar provides a range of environments from aerobic to anaerobic and permits the suspended inoculum to develop in that region of the medium most suitable for it. With this soft agar medium it is possible to determine when the utilization of an energy source is only the property of a mutant population, and whether growth with a particular substrate is aerobic only, facultative, anaerobic only, or microaerophilic. It was also possible to observe the special effects of temperature of incubation, $\mathrm{pH}$ value, sodium chloride concentration, the method of sterilizing energy sources, and the addition to the medium of citrate, manganese and other substances. Liquid media incubated aerobically are much less informative; they tended to give variable results when restricted conditions were required for substrate utilization or when growth was dependent upon a mutation. A question which was not investigated is the effect of the $\mathrm{E}_{h}$ value at different depths of the soft agar medium. Some of the observations concerning growth at particular regions in the medium might indicate differences in $\mathrm{E}_{h}$ requirement by the given organisms. It is evident from the observations made with soft agar cultures that it is not possible to generalize about the oxygen requirements of the lactic acid bacteria. The present survey has indicated that differences in the requirements are especially numerous among leuconostocs related to Leuconostoc mesenteroides and among heterofermentative lactobacilli classified as Lactobacillus brevis, L. buchneri and $L$. viridescens. 
The results obtained with soft agar could provide starting points for biochemical investigations as indicated by the following two examples. The first concerns the unusual behaviour of a leuconostoc which grows and ferments sugars only when oxygen is available. Growing cultures form $\mathrm{H}_{2} \mathrm{O}_{2}$ and cell-suspensions rapidly take up oxygen in presence of glucose and form $\mathrm{H}_{2} \mathrm{O}_{2}$. This suggests $(a)$ that flavoprotein oxidases play a significant role in the successful growth of this organism and $(b)$ that one or more of the dehydrogenases which might be expected to function in anaerobic fermentation are failing to do so or are not being formed. The second case concerns the strictly aerobic growth of some strains of Lactobacillus brevis and L. buchneri with certain separately sterilized substrates (see Table 1). It may be suggested that one or more of the dehydrogenases which operate in an anaerobic fermentation of gluconate, glucose and related compounds are not functioning or are not being formed when the substrate has been sterilized separately. The rapid development of aerobic growth suggests that flavoprotein oxidases and/or peroxidases may function in place of the missing anaerobic dehydrogenase(s), oxygen being used as a hydrogen acceptor.

The author wishes to thank Dr T. Gibson for advice and encouragement given during this work and for helpful criticism given during preparation of the manuscript, and Dr M. Elizabeth Sharpe and Dr P. M. Frances Shattock for providing some of the strains studied.

\section{REFERENCES}

Bergey's Manual of Determinative Bacteriology (1957). 7th edn. Ed. by R. S. Breed, E. G. D. Murray and N. R. Smith. Baltimore: The Williams and Wilkins Co.

Dobrogosz, W. J. \& Stone, R. W. (1959). Glycerol metabolism of pediococci isolated from alfalfa silages. Bact. Proc. p. 111.

Gibson, T., Stirling, A. C., Keddie, R. M. \& Rosenberger, R. F. (1958). Bacteriological changes in silage made at controlled temperatures. J. gen. Microbiol. 19, 112.

Gunsalus, I. C. \& Sherman, J. M. (1943). The fermentation of glycerol by streptococci. J. Bact. 45, 155.

Kneteman, A. (1947). A reaction on the formation of hydrogen peroxide by microorganisms in solid media. Antonie van Leeuwenhoek J. Microbiol. Serol. 13, 55.

Nakagawa, A. \& Kitahara, K. (1959). Taxonomic studies on the genus Pediococcus. J. gen. appl. Microbiol. 5, 95.

Rogosa, M., Wiseman, R. F., Mitchell, J. A., Disraely, M. N. \& Beaman, A. J. (1953). Species differentiation of oral lactobacilli from man including descriptions of Lactobacillus salivarius nov.spec. and Lactobacillus cellobiosus nov.spec. J. Bact. 65, 681.

\section{EXPLANATION OF PLATES}

The growth of lactic acid bacteria at $30^{\circ}$ in soft agar contained in $6 \times \frac{5}{8}$ in. test tubes.

Plate 1

Fig. 1. A strain of Streptococcus faecium utilizing glycerol aerobically; $48 \mathrm{hr}$.

Fig. 2. A strain of the Lactobacillus plantarum-casei group utilizing cellobiose anaerobically; $72 \mathrm{hr}$.

Fig. 3. L. cellobiosus utilizing glucose anaerobically; $24 \mathrm{hr}$.

Fig. 4. L. cellobiosus utilizing glucose; growth beginning to develop in upper part of the medium; $36 \mathrm{hr}$.

Fig. 5. Pediococcus NCDo 1250 utilizing glucose anaerobically; $48 \mathrm{hr}$.

Fig. 6. A strain of $L$. buchneri utilizing xylose microaerophilically; $72 \mathrm{hr}$.

Fig. 7. A strain of $L$. fermenti utilizing glucose equally well aerobically and anaerobically; $24 \mathrm{hr}$. 
Fig. 8. A strain of $S$. faecium utilizing sorbitol aerobically and anaerobically after mutation. $A$ haze of non-acid-forming growth surrounds mutant colonies; 6 days.

Fig. 9. A strain of pediococcus utilizing lactose mutantly. A haze of non-acid-forming growth surrounds colonies; 5 days.

Fig. 10. A strain of the $L$. plantarum-casei group utilizing cellobiose mutantly. A haze of nonacid-forming growth surrounds colonies; 5 days.

Fig. 11. A strain of the $L$. plantarum-casei group utilizing sucrose aerobically but mutantly; 4 days.

Fig. 12. A strain of the L. plantarum-casei group utilizing cellobiose anaerobically but mutantly; 5 days.

Fig. 13. A strain of the L. plantarum-casei group utilizing mannitol mutantly. Sub-surface disk of growth not acid-producing, but acid-forming colonies developed from this disk of growth; 7 days. Fig. 14. A strain of the $L$. plantarum-casei group; a single mutant sucrose-utilizing colony developing; 7 days.

Plate 2

Fig. 15. Pediococcus soyae ram 1673 utilizing glucose in the presence of $5 \%(\mathrm{w} / \mathrm{v}) \mathrm{NaCl} ; 4$ days.

Fig. 16. $P$. soyae IAM 1673 utilizing glucose in the presence of $15 \%(\mathrm{w} / \mathrm{v}) \mathrm{NaCl} ; 4$ days.

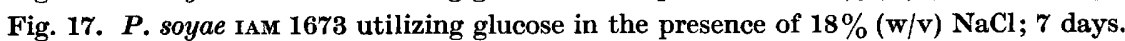

Fig. 18. A strain of pediococcus utilizing glucose in the presence of $10 \%(\mathrm{w} / \mathrm{v}) \mathrm{NaCl} ; 5$ days.

Fig. 19. Lactobacillus fructovorans NCIB 8039, growth distribution in glucose soft agar; 3 days at $35^{\circ}$.

Fig. 20. L. fructovorans NсIв 8039, growth distribution in glucose soft agar; 3 days at $37^{\circ}$.

Fig. 21. A non-dextran-forming leuconostoc utilizing glucose; 3 days. Aerobic (surface) growth is separated from the anaerobic growth by a growth-free zone. Inhibition of growth shown to be caused by $\mathrm{H}_{2} \mathrm{O}_{2}$ diffusing downwards from the aerobic growth.

Fig. 22. As fig. 21, 5 days. The growth-free zone has diminished on continued incubation. 

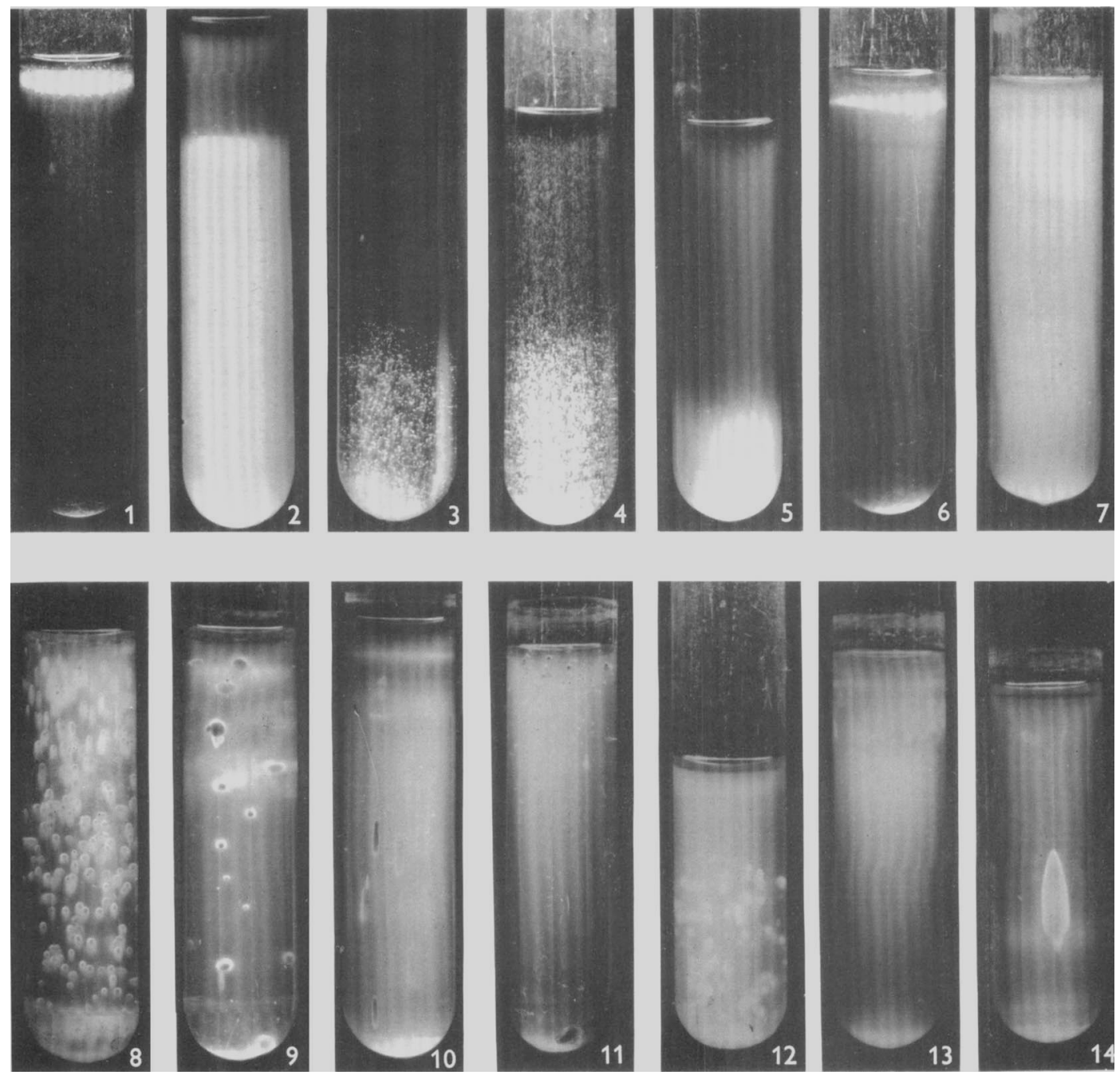

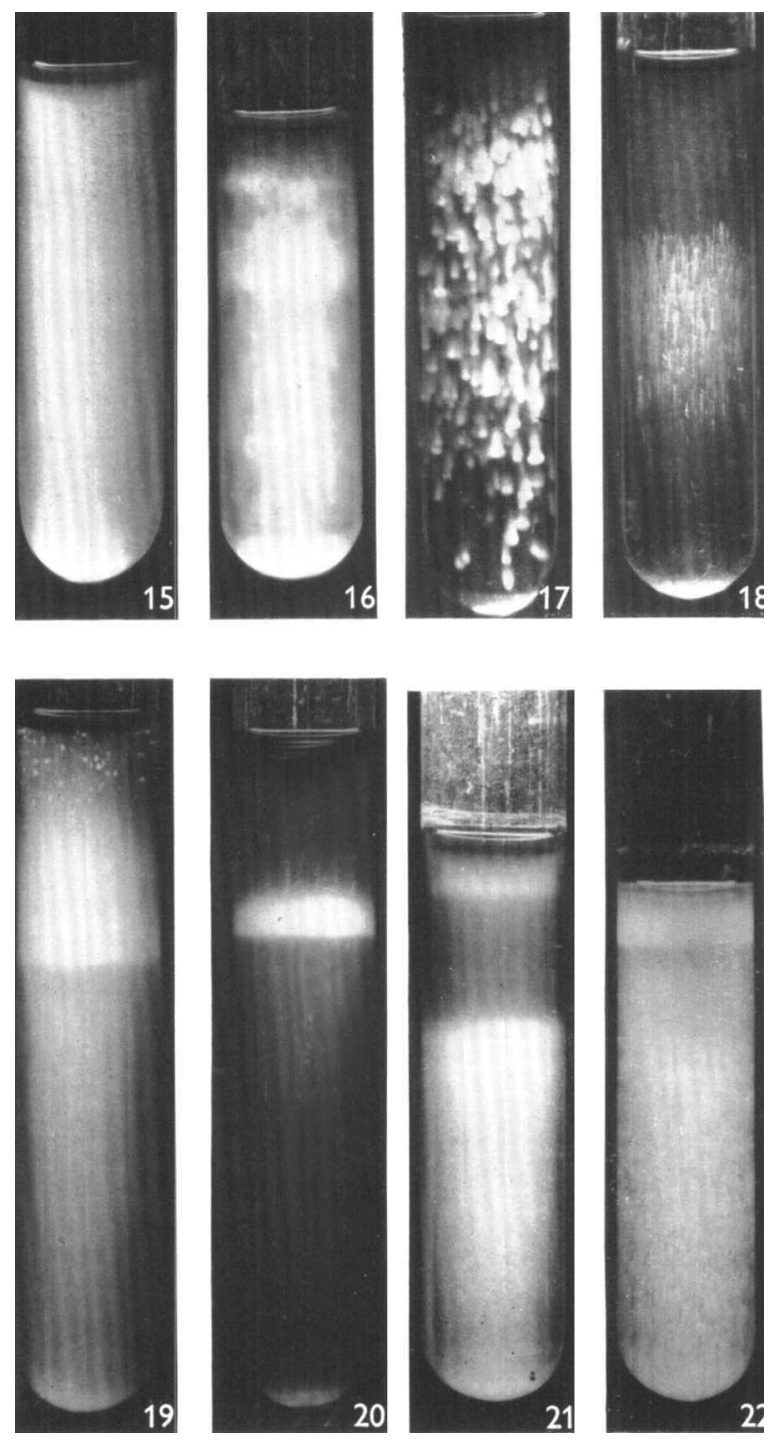

R. WHITTENBURY 